\title{
La voce del paziente: accesso alla lista d'attesa e post trapianto: raccontaci la tua testimonianza. . .
}

Giornale di Tecniche Nefrologiche e Dialitiche 2019, Vol. 3I(3) 183-185

(C) The Author(s) 2019

Article reuse guidelines:

sagepub.com/journals-permissions DOI: $10.1177 / 0394936219869169$ journals.sagepub.com/home/gtn

(S)AGE

\section{Luisa Sternfeld Pavia}

$\checkmark$ Conduco una vita abbastanza tranquilla dopo il trapianto perché non ho più la preoccupazione di dover fare la dialisi. Prima del trapianto non vivevo bene la mia malattia, ma sono stata abbastanza metodica nel seguire ogni prescrizione per ritardare l'ingresso in dialisi e al quinto stadio IRC, essendo in buone condizioni fisiche, ancora non avevo sintomi di crisi uremica e mi è stato consigliato di iscrivermi in lista d'attesa per il trapianto pre-emptive. Dopo 11 mesi sono stata chiamata e ora sto bene.

$\checkmark$ Ho solo dei suggerimenti per migliorare la vita del paziente trapiantato: fornire lista di farmaci che si possono assumere esempio per influenze, infezioni gastrointestinale, supporto psicologico post trapianto. Chiarire meglio $\mathrm{i}$ tempi di verifica dosaggio immunosoppressori. Ho fatto trapianto da donatore cadavere e mi sono trovata bene.

$\checkmark$ Il trapianto è l'esperienza emotivamente più bella e complessa che abbia mai vissuto. La rinascita fisica è stata poi la ciliegina sulla torta. È valsa la pena affrontare le sofferenze fisiche ed emotive della dialisi (e della nefrectomia) perché queste tappe, seppur faticose, mi hanno permesso di vivere la rinascita. $\mathrm{Mi}$ sento più fortunata dei miei coetanei perché la dialisi mi ha tolto e mi ha dato tanto. Mi ha permesso di mettere in ordine la mia vita e di dare il giusto peso alle cose. Mi ha fatto capire che l'amore e la generosità sono capaci di superare qualsiasi barriera. Tutto questo è stato possibile anche grazie ad un grande staff che mi ha sempre seguita con professionalità ed affetto.

Il trapianto è senz'altro un dono grazie alla sensibilità di coloro che dispongono in tal senso e sarò sempre grata per quello che ho ricevuto. Tuttavia devo segnalare alcuni limiti. La terapia immunosoppressiva è pesante! Gli effetti collaterali sono molteplici e poco considerati dai medici che probabilmente temono che il paziente nel lungo periodo perda la costanza nella terapia. Il limite più evidente e fastidioso è la perdita costante di energia anche quando la terapia viene ridotta e la sensazione di essere diversi da prima che è inevitabile, sarebbe strano il contrario, ma che influenza anche il rapporto di coppia. Ho trovato utile l'incontro con uno psicologo che mi ha permesso post trapianto di liberare il dolore e la scrittura terapeutica guidata per lo stesso motivo. Ci si concentra molto sull'arrivare in buone condizioni al trapianto (io avevo 44 anni) ma è altrettanto importante il seguito!

$\checkmark$ Vita lavorativa e sociale normale, ho ripreso gli studi e mi sono laureato.

La qualità della vita dopo il trapianto non è più la stessa. Gli effetti collaterali dei farmaci impediscono una vita normale. Certamente, dopo la dialisi, ringrazio comunque Dio tutti i giorni per il trapianto.

$\checkmark$ Io sono stato fortunato nel senso che il donatore è mia moglie. Non ho mai fatto dialisi e il decorso post trapianto è regolarissimo. Assieme a mia moglie facciamo sport (palestra, sci) e giardinaggio full immersion. Pensavo di fare l'invalido. . . invece ora ho 73 anni e sono 11 anni dall'intervento e se continua così ci faccio la firma!!!

Il 28 giugno festeggio i miei primi 25 anni dal trapianto di rene. Sono rinata.

Ho avuto 2 trapianti, il primo dopo 9 mesi di dialisi, il secondo dopo 12 anni. Sono passati 9 anni dall'ultimo

AIRP onlus, Milan, Italy

Corrispondenza:

Luisa Sternfeld Pavia, AIRP onlus, Via Bazzini, 2, Milano 2013I, Italy.

E-mail: luisa.sternfeld.airp@renepolicistico.it 
trapianto, attualmente sto bene, i dati delle analisi sono positivi, consiglio a tutti grande attenzione nel primo anno dopo il trapianto e rigorosa attenzione alla terapia.

$\checkmark$ In poche righe è difficile. . ma GRAZIE per quello che fate!

$\checkmark$ Buongiorno. .. poche righe è veramente difficile. Nel maggio 2015 faccio un prelievo perché vorrei perdere qualche kg post gravidanze ed invece scopro insufficienza renale (totalmente asintomatica). Mi rivolgo ad un nefrologo e la diagnosi è RENE POLICISTICO. Dialisi e trapianto ci cade il mondo. . . Inizia la mia storia, la nostra storia: sono figlia, moglie e mamma. Si inizia con una dieta aproteica, nel frattempo mi viene confezionata la fistola al braccio sx per iniziare la dialisi che poi inizierò il 5 dicembre 2015. Il mio centro di riferimento mi indica la possibilità di essere ricoverata per esami compatibilità con mio marito. . . si fa! Ci hanno scrutato ogni mm del nostro corpo. . . siamo compatibili!!! Nel frattempo ho una colica epatica. . . ulteriori esami. . diagnosi: policistosi epato renale. Mi consigliano trapianto combinato fegato rene. . salta tutto... Ricado nel dolore profondo... dimenticavo che per conquistare una cosa serve pazienza e sacrificio. . Si inizia 5 dicembre accolta da un team preparato e di un umanità indescrivibile. . . continuo a fare controlli per entrare in lista attiva. Il 5 aprile 2016 firmo tutti i documenti necessari sono iscritta definitivamente. La mia vita continua tra lavoro, dialisi e famiglia; riusciamo a fare un paio di week-end non troppo lontano da casa. . la valigia pronta come mi hanno detto al centro trapianto. In ottobre nella mia parrocchia arriva la Madonna Pellegrina e affido a Lei le mie fatiche. . Arriva dicembre mese intenso di lavoro dato che faccio commessa in un negozio di giochi, dializzo ancora 2 volte a settimana, a metà dicembre i medici mi dicono che dovrò iniziare a fare anche la terza dialisi. . . il 25 e 26 si festeggia in famiglia. Il 26 dicembre 2016 ore 16.25 ricevo la telefonata che tanto attendevo: PROBABILE TRAPIANTO! Il 27 dicembre 2016 ore 5.00 entro in sala operatoria!!! Inizia una nuova storia. . . grazie ad un ANGELO che vive lassù, so che mi guarda. . . io faccio di tutto per proteggere e custodire caro il DONO ricevuto. Non conosco chi sono i suoi famigliari ma li ricordo e li affido. Questi 2 anni? Tosti! Non ho avuto un trapianto, ma guai lamentarsi. . . se ci sarà occasione sarò dettagliata. Grazie di cuore per tutto quello che fate!!!

La mia esperienza è molto positiva. Sono stata seguita da infermieri e medici competenti.
Il problema più grosso dal punto di vista della gestione del paziente l'ho trovato nell'immediato post trapianto perché non sentivo di essere seguita con attenzione visto che il medico di base e i medici del centro trapianti locale rimandavano le mie cure all'ospedale dove era stato eseguito il trapianto, dove non sempre si riesce ad avere risposte in tempi celeri e spesso c'è un rimbalzo di responsabilità.

$\checkmark$ Dopo 15 giorni dal trapianto da vivente (donatore mio marito) ho avuto un meningioma operato durante la degenza post trapianto. Ho subito la nefrectomia PRIMA del trapianto; dopo un paio d'anni dal trapianto sono di molto aumentate le cisti al fegato ora molto voluminoso. Ho avuto un melanoma operato a 4 anni dal trapianto ora completamente guarito.

In famiglia ho avuto padre 12 anni di dialisi (anni $70 / 80$ ) deceduto a 53 anni la sorella di mio padre emodialisi e trapianto in Belgio (1980) con rigetto subito e deceduta dopo pochi mesi a 43 anni. Ho passato l'infanzia contornata dalla dialisi e ho sempre saputo di essere una bomba ad orologeria. . . a 53 anni (2013) iniziato dialisi peritoneale notturna. Avevo paura ad entrare in lista, rimandavo trovavo delle scuse. . . Poi essendo seguita da una nefrologa molto competente e anche molto sensibile, mi ha accompagnato in questa grande decisione (lo deve alle sue figlie, la medicina in tanti anni è andata avanti ecc.). Entrata in lista e dopo sei mesi la chiamata. . ora sono 2 anni e se tornassi indietro mi riprenderei la mia vita di corsa. Buona vita a tutti!

Esperienza difficile per motivi di salute legati alla mia patologia. Esperienza positiva per quanto riguarda il centro trapianto!

$\checkmark$ Dopo il buio arriverà la luce e così è stato.

Nel giro di 2 anni ho iniziato dialisi, tolto rene dx, arrivato trapianto, tolto rene sx. Ci vuole tanta pazienza sia da dializzato che da trapiantato.

Primi mesi rinata, ora stressata ma sempre una benedizione, senza il trapianto credo avrei vissuto peggio. Grazie a tutti i nostri angeli donatori.

La sanità italiana non è organizzata per seguire trapiantati con problemi collegati alle terapie. Solo follow-up nefrologico.

Io sono un trapiantato da donatore vivente (mia moglie) e la qualità della vita è sicuramente migliorata. Posso 
fare tutto con attenzione ma nessuna negazione. Unico inconveniente la presenza e la necessità di asportazione di cheratosi atomiche.

Trapiantato a Sassari, sono stato seguito ottimamente sia nel pre che nel post-trapianto. Oramai sono quasi 16 anni dal trapianto, la mia vita è proseguita, se non proprio normale, almeno in maniera accettabile. Ho avuto una figlia alla quale purtroppo è stato diagnosticato il rene policistico bilaterale e questo è il cruccio maggiore della mia vita. Aver rischiato sapendo che avevamo il $50 \%$ di possibilità. . .

$\checkmark$ Grazie a Dio mi sono liberata della dialisi! La dialisi è un incubo, uscirne è stato meraviglioso.

Sono sei mesi che ho ricevuto un rene, un dono grandissimo sono grato e felice.

La mia esperienza sino a qui è sicuramente positiva. L'assunzione regolare dei farmaci comporta diversi effetti collaterali che hanno come conseguenza diretta quella di complicare la vita di tutti i giorni e il modo di relazionarsi con il prossimo. Mi riferisco in particolare al cortisone che oltre a causare effetti collaterali diretti come gastrite etc. provoca sbalzi di umore che creano difficoltà come già citato nella gestione delle relazioni umane. In ogni caso il trapianto dà la possibilità a persone che soffrono e che si sentono ormai ai margini di riappropriarsi della propria vita ridando loro dignità e nuovi stimoli.

È stato un percorso non facile ma nel mio cammino sia prima e post trapianto, ho trovato delle persone adorabili sia medici che personale paramedico.

$\checkmark$ Il miglioramento della vita dopo il trapianto, soprattutto niente più dialisi, mi fa accettare con più serenità anche le "complicazioni" che sono venute.

$\checkmark$ Mi sento una persona normalissima senza nessun problema, ho ripreso con le gare di ciclismo dopo 35 anni, domenica 14/04/2019 ho fatto una gara e sono arrivato secondo con alcuni avversari dell'epoca, e tanti più giovani di 15 anni.

$\checkmark$ Grazie al trapianto la mia vita sociale e familiare è cambiata in modo positivo. Ho avuto, e forse ne avrò ancora, problemi dovuti ai farmaci, ma grazie alla mia forza di volontà e alla mia famiglia sono riuscito a superarli. Grazie al mio donatore

Ho fatto dialisi per 3 anni, dopo 13 mesi ho fatto binefrectomia e una volta in lista ho aspettato solo 17 mesi per ricevere il rene. Con grande gioia ringrazierò sempre il mio donatore. 